\title{
Dysphagia in Patients with Acute Ischemic Stroke: Early Dysphagia Screening May Reduce Stroke-Related Pneumonia and Improve Stroke Outcomes
}

\author{
Mohamed Al-Khaled ${ }^{a} \quad$ Christine Matthis $^{\mathrm{b}} \quad$ Andreas Binder $^{\mathrm{d}} \quad$ Jonas Mudter $^{\mathrm{e}}$ \\ Joern Schattschneider ${ }^{f} \quad$ Ulrich Pulkowskig ${ }^{\text {Tim Strohmaier }^{\mathrm{h}}}$ Torsten Niehoff $^{\mathrm{i}}$ \\ Roland Zyburj Juergen Eggers ${ }^{c}$ Jose M. Valduezak ${ }^{k}$ Georg Royl ${ }^{a}$ \\ for QugSS II Group

\begin{abstract}
${ }^{a}$ Department of Neurology and ${ }^{b}$ Institute of Social Medicine, University of Lübeck, and ${ }^{\mathrm{C}}$ Department of Neurology, Sana Hospital Lübeck, Lübeck, d Department of Neurology, University of Kiel, Kiel, e Department of Internal Medicine and Stroke Unit, Sana Hospitals Eutin, Eutin, fDepartment of Neurology, Ev.-Luth. Diakonissenanstalt Flensburg, Flensburg, ${ }^{9}$ Department of Neurology, Imland Hospital Rendsburg, Rendsburg, h' Department of Neurology, Friedrich-Ebert Hospital, Neumünster, 'Department of Neurology, Sana-Hospital Pinneberg, Pinneberg, jDepartment of Neurology, Westkuesten-Hospital, Heide, and kDepartment of Neurology, Segeberger Hospitals, Bad Segeberg, Germany
\end{abstract}

\section{Key Words}

Stroke - Dysphagia - Dysphagia screening · Pneumonia .

Outcome $\cdot$ Mortality

\begin{abstract}
Background: Dysphagia is associated with poor outcome in stroke patients. Studies investigating the association of dysphagia and early dysphagia screening (EDS) with outcomes in patients with acute ischemic stroke (AIS) are rare. The aims of our study are to investigate the association of dysphagia and EDS within $24 \mathrm{~h}$ with stroke-related pneumonia and outcomes. Methods: Over a 4.5-year period (starting November 2007), all consecutive AIS patients from 15 hospitals in Schleswig-Holstein, Germany, were prospectively evaluated. The primary outcomes were stroke-related pneumonia
\end{abstract}

\section{KARGER}

E-Mail karger@karger.com www.karger.com/ced during hospitalization, mortality, and disability measured on the modified Rankin Scale $\geq 2-5$, in which 2 indicates an independence/slight disability to 5 severe disability. Results: Of 12,276 patients (mean age $73 \pm 13 ; 49 \%$ women), 9,164 patients (74\%) underwent dysphagia screening; of these patients, $55,39,4.7$, and $1.5 \%$ of patients had been screened for dysphagia within 3, 3 to $<24,24$ to $\leq 72$, and $>72$ h following admission. Patients who underwent dysphagia screening were likely to be older, more affected on the National Institutes of Health Stroke Scale score, and to have higher rates of neurological symptoms and risk factors than patients who were not screened. A total of 3,083 patients $(25.1 \% ; 95 \% \mathrm{Cl}$ 24.4-25.8) had dysphagia. The frequency of dysphagia was higher in patients who had undergone dysphagia screening than in those who had not (30 vs. $11.1 \%$; $p<0.001$ ). During hospitalization (mean 9 days), 1,271 patients $(10.2 \% ; 95 \% \mathrm{Cl}$
(C) 2016 S. Karger AG, Basel

$1015-9770 / 16 / 0422-0081 \$ 39.50 / 0$
PD Dr. Mohamed Al-Khaled

Department of Neurology

University of Lübeck, Ratzeburger Allee 160

DE-23538 Lübeck (Germany)

E-Mail mohamed.al-khaled@ neuro.uni-luebeck.de 
9.7-10.8) suffered from stroke-related pneumonia. Patients with dysphagia had a higher rate of pneumonia than those without dysphagia (29.7 vs. $3.7 \% ; p<0.001$ ). Logistic regression revealed that dysphagia was associated with increased risk of stroke-related pneumonia (OR 3.4; 95\% Cl 2.8-4.2; $\mathrm{p}<$ $0.001)$, case fatality during hospitalization (OR $2.8 ; 95 \% \mathrm{Cl}$ 2.1-3.7; $p<0.001)$ and disability at discharge (OR 2.0;95\% Cl $1.6-2.3 ; \mathrm{p}<0.001)$. EDS within $24 \mathrm{~h}$ of admission appeared to be associated with decreased risk of stroke-related pneumonia (OR $0.68 ; 95 \% \mathrm{Cl} 0.52-0.89 ; \mathrm{p}=0.006)$ and disability at discharge (OR $0.60 ; 95 \% \mathrm{Cl} 0.46-0.77 ; \mathrm{p}<0.001$ ). Furthermore, dysphagia was independently correlated with an increase in mortality (OR $3.2 ; 95 \% \mathrm{Cl} 2.4-4.2 ; \mathrm{p}<0.001)$ and disability (OR $2.3 ; 95 \% \mathrm{Cl} 1.8-3.0 ; \mathrm{p}<0.001)$ at 3 months after stroke. The rate of 3-month disability was lower in patients who had received EDS ( 52 vs. $40.7 \% ; p=0.003$ ), albeit an association in the logistic regression was not found (OR 0.78; $95 \% \mathrm{Cl} 0.51-1.2 ; \mathrm{p}=0.2)$. Conclusions: Dysphagia exposes stroke patients to a higher risk of pneumonia, disability, and death, whereas an EDS seems to be associated with reduced risk of stroke-related pneumonia and disability.

(c) 2016 S. Karger AG, Basel

\section{Introduction}

Dysphagia is a common neurological symptom of stroke and portends a higher risk of complication after stroke, particularly pneumonia caused by the dysfunction of cough reflex and aspiration and advanced by stroke-induced immunodepression [1,2]. Studies have shown that dysphagia affects up to two thirds of patients with stroke [3-5], depending on the stroke's type, size, site and severity. Furthermore, the occurrence of dysphagia in patients with stroke has been linked to poor outcomes with higher risks for pneumonia, mortality and disability $[3,5]$. Stroke-related pneumonia has been found to be correlated with longer periods of hospitalization and an increase in the financial costs of the medical care of stroke patients [6]. Studies have found that early aspiration prophylaxis using tube feeding after stroke may reduce case fatality; however, early feeding of stroke patients with dysphagia by percutaneous endoscopic gastrostomy (PEG) was not supported [7]. Stroke guidelines recommend that early dysphagia screening (EDS) be performed in stroke patients to detect swallowing difficulties and to prevent stroke-related pneumonia caused by aspiration combined with impairment of humeral and neural systems $[6,8]$. Although there is no doubt about the relevance of dysphagia in acute ischemic stroke (AIS), large-scale quantitative data on stroke-associated dysphagia and pneumonia in large cohort are lacking. With this prospectively designed hospital-based study, we aimed to study the association between dysphagia as well as EDS within $24 \mathrm{~h}$ of admission with the occurrence of stroke-related pneumonia and outcome.

\section{Methods}

\section{Study Design}

From November 2007 through March 2012, a total of 12,781 patients (mean age $73 \pm 13 ; 48.6 \%$ women; median National Institutes of Health Stroke Scale (NIHSS) score, 4 (interquartile range (IQR) 2-9)) with AIS were included in this hospital-based study. The study is a part of the ongoing stroke registry, Quality of Treatment of Stroke in the Federal State Schleswig-Holstein (in German, Qualitätsgemeinschaft Schlaganfallversorgung in Schleswig-Holstein (QugSS2)) as benchmarking project to improve stroke care in Schleswig-Holstein. The project (QugSS2), which has been previously described [9], includes all 15 hospitals involved in the treatment of patients with stroke in the German federal state of Schleswig-Holstein, which has 2.8 million inhabitants. The stroke care among included hospitals is standardized as recommended by the German Stroke Society and German Association of Neurology. The inclusion criterion of the study were that the patients had to be residents of the state of Schleswig-Holstein with a diagnosis of AIS. The diagnosis of stroke was based on clinical presentation and brain imaging (cranial CT and MRI). Documentation and datacollection procedures that we conducted followed a uniform study manual as part of a benchmarking project.

\section{Data Acquisition}

Data acquisition was performed prospectively during hospitalization from patient records. Medical histories or prior records were also included in the documentation of the data. The study protocol was placed in the individual file of each patient. The treating physicians filled in the baseline characteristics at admission and completed the protocol at discharge. Patients with AIS (age $\geq 18$ years) whose main residence was in the German federal state of Schleswig-Holstein were included. Stroke patients who presented to the emergency department but declined hospital admission were not included in the stroke registry. Patients were also excluded from the study if they were admitted with suspected AIS but were given a different diagnosis after undergoing diagnostic evaluation during hospitalization. Baseline characterizations including gender, age, modified Rankin Scale (mRS) score, NIHSS score, medical history, treatments of stroke, secondary prevention strategies, and etiology of the stroke in accordance with the Trial of Org 10172 in Acute Stroke Treatment (TOAST) classification were recorded [10]. The case fatality was defined as mortality during hospitalization, whereas disability as $m R S \geq 2-5$ at discharge and after 3 months.

\section{Follow-Up Evaluation}

At discharge, stroke patients or caregivers were asked whether they agreed to be included in the follow-up questionnaire that was conducted 3 months after discharge. Patients were first questioned
Al-Khaled et al. 
by letter. Those patients who did not respond to the letter were then contacted by telephone. If they were not available, we evaluated mortality at 3 months after discharge by sending an online request to the registration office of the German federal state of Schleswig-Holstein. If discharged patients were no longer residing in the state of Schleswig-Holstein, they were considered lost to follow-up.

\section{Dysphagia Screening}

In accordance with the stroke unit care and as part of the stroke registry protocol, every stroke patient should undergo a dysphagia screening at admission as soon as possible regardless of the neurological symptoms and whether a dysphagia was suspected or not. Nurses or treating physicians performed systematic dysphagia screening clinically on admitted patients before feeding or administration of oral drugs. Water and/or thickened apple juice were used to test whether swallowing abnormalities were evident. In addition, swallowing and cough provocation assessments were performed using a thin tube inserted into the oropharynx area to test the sensory input and motor output in the pharynx area. With this procedure, the delay or lack of the initiation of the swallowing process can be assessed in order to detect the silent aspiration. Dysphagia was determined in cases of deglutition, drooling, absent swallow reflex, cough or voice change after swallowing, reduced water control, decreased oral clearance, or involuntary cough. If swallowing difficulties were suspected, swallowing therapists repeated the dysphagia screening and possibly performed further dysphagia tests; in addition, measures of swallowing therapy were initiated. In cases with evidence of dysphagia after hospital admission, initial feeding and the administration of drugs were carried out through peripheral or/and central venous catheter or by nasogastral tubes. When dysphagia was persistent during the hospitalization, PEG was generally initiated before patients were discharged or during the rehabilitations period.

\section{Pneumonia}

In accordance with the criteria used in the literature to diagnose pneumonia after stroke, although a wide range of definition exists [6], the diagnosis in this study was based on a combination of clinical presentations, radiologic signs detected on a chest X-ray, and blood test results (C-reactive protein and leukocytes).

Standard Protocol Approval, Registration, and Patient Consent Approval for the study was obtained from the local Ethics Committee of the University of Lübeck. Entry in the stroke registry was obligatory as part of the benchmarking project to improve stroke care. At the time of discharge from the hospital, stroke patients or caregivers were asked if they could be included in the follow-up evaluation conducted 3 months after discharge from the hospital. If they agreed to participate in the follow-up evaluation, an informed consent form was obtained from them.

\section{Statistics}

The SPSS program (version 22; IBM SPSS Statistics, Armonk, N.Y., USA) was used to analyze data. We described with mean and SD values for continuous variables, median and IQR values for scores, and absolute numbers and percentages for nominal and categorical variables. We performed a chi-square test to determine the correlation between categorical variables, a t test between continuous variables, and a Mann-Whitney test between scores. Logistic regression was carried out to estimate the OR. Variables with a p value $<0.1$ were entered into the logistic regression model. A p value $<0.05$ was considered significant. In cases of multiple comparisons, a Bonferroni correction was used to show whether the individual comparison was significant or not.

\section{Results}

\section{Dysphagia}

During a 4.5-year study period (starting November 2007), 12,781 patients with AIS were admitted to 15 hospitals. Most patients (87\%) were admitted within $6 \mathrm{~h}$ of symptom onset. The time point of dysphagia screening was documented for $74 \%$ of patients $(n=9,164)$; of these patients, $55,39,4.7$, and $1.5 \%$ of patients had been screened for dysphagia within 3,3 to $<24,24$ to $\leq 72$, and $>72 \mathrm{~h}$ following admission. The rates of altered consciousness were $9.7,11.5,16.5$ and $26.9 \%$ in patients with AIS who were screened for dysphagia within 3, 3 to $<24$, 24 to $\leq 72$, and $>72$ h of admission.

A comparison between patients who underwent dysphagia screening and those who were not screened is shown in table 1 as well as a comparison between patients who underwent an EDS within 24 vs. those who screened later is shown in table 1.

For 12,276 patients (96\%), data on the presence of dysphagia were available. Of these patients, 3,083 patients (25.1\%; 95\% CI 24.4-25.8) had dysphagia.

The dysphagia rates were $27.5,31.7,41.8$, and $42.3 \%$ in patients who had undergone dysphagia screening within 3,3 to $<24,24$ to $\leq 72$, and $>72 \mathrm{~h}$ following hospital admission, respectively (fig. 1). When pooling into 2 groups ( $<24$ vs. $\geq 24 \mathrm{~h}$ ), dysphagia incidence was significantly increased among patients who were screened after $24 \mathrm{~h}$ of admission compared to those who underwent an EDS within $24 \mathrm{~h}$ of admission ( 42 vs. $29 \%$; $\mathrm{p}<0.001$ ). As shown in table 2, AIS patients with dysphagia were more likely to be older (76 vs. 72 years, respectively; $p<0.001$ ) and to have a higher NIHSS score at admission ( 13 vs. 3 , respectively; $\mathrm{p}<0.001$ ) than those without dysphagia. They also had a higher probability of altered consciousness at admission and speech dysfunction than patients without dysphagia.

\section{Stroke-Related Pneumonia and Outcomes}

A total of 1,271 AIS patients (10.2\%; 95\% CI 9.7-10.8) had stroke-related pneumonia. A comparison of patients with dysphagia and those without dysphagia revealed that patients with dysphagia had higher rates of pneumonia ( 29.7 vs. $3.7 \%$, respectively; $\mathrm{p}<0.001$ ); case fatality 
Table 1. Comparison between patients who underwent a dysphagia screening vs. those who did not and between patients who had EDS done within $24 \mathrm{~h}$ vs. delayed dysphagia screening

\begin{tabular}{|c|c|c|c|c|c|c|}
\hline Baseline characteristics & \multicolumn{2}{|c|}{ Dysphagia screening } & $\mathrm{p}$ value & \multicolumn{2}{|c|}{ EDS within $24 \mathrm{~h}$} & $\mathrm{p}$ value \\
\hline Median NIHSS score (IQR) & $3(1-3)$ & $4(2-10)$ & $<0.001$ & $7(3-15)$ & $4(2-9)$ & $<0.001$ \\
\hline Sex, male & $1,657(52)$ & $4,661(51)$ & 0.1 & $268(48)$ & $4,393(51)$ & 0.1 \\
\hline Altered consciousness & $245(9)$ & $992(11)$ & $<0.001$ & $105(19)$ & $887(10)$ & $<0.001$ \\
\hline Hypertension & $2,562(82)$ & $7,645(85)$ & $<0.001$ & $446(81)$ & $7,199(85)$ & 0.008 \\
\hline Diabetes mellitus & $737(24)$ & $2,377(27)$ & 0.001 & $144(26)$ & $2,233(27)$ & 0.7 \\
\hline Hypercholesterolemia & $1,593(52)$ & $4,890(56)$ & 0.001 & $242(45)$ & $4,648(56)$ & $<0.001$ \\
\hline Previous stroke & $837(27)$ & $2,648(30)$ & 0.003 & $151(27)$ & $2,497(30)$ & 0.2 \\
\hline Atrial fibrillation & $794(26)$ & $2,972(33)$ & $<0.001$ & $190(34)$ & $2,782(33)$ & 0.6 \\
\hline AT before stroke & $1,240(40)$ & $3,900(44)$ & $<0.001$ & $235(43)$ & 3,665 (44) & 0.8 \\
\hline
\end{tabular}

Data are $\mathrm{n}(\%)$ unless otherwise indicated. AT = Antiplatelet therapy.

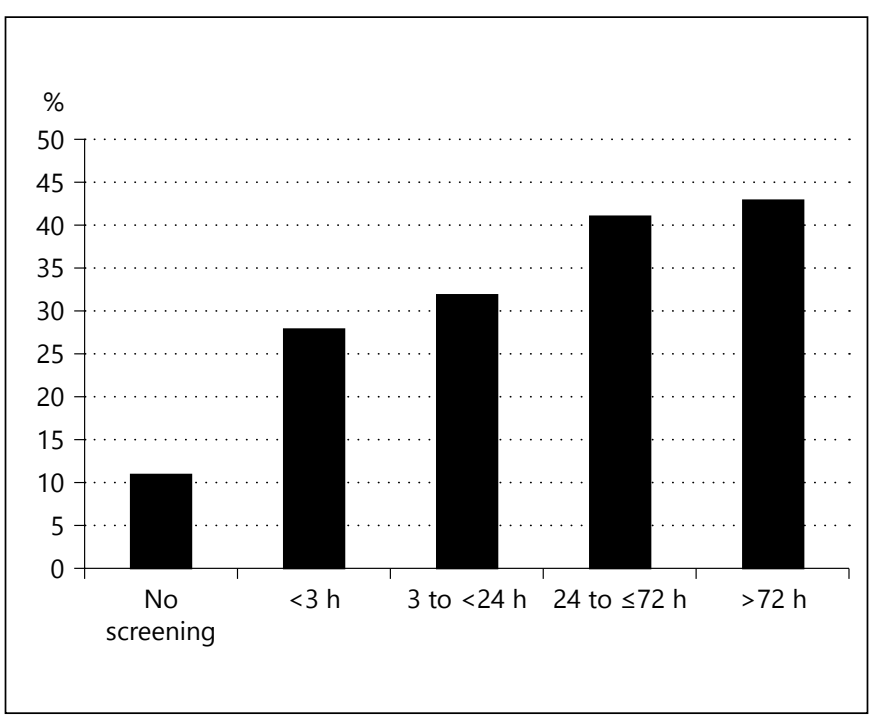

Fig. 1. Dyspaghia frequency in relation to time point of dysphagia screening. $\mathrm{X}$-axis indicates time point of dysphagia screening, $\mathrm{Y}$ axis indicates frequency of dysphagia.

(14.9 vs. $1.2 \%$, respectively; $\mathrm{p}<0.001)$ and a longer hospitalization in acute care $(10.5$ vs. 9 days; $\mathrm{p}<0.001)$. Pneumonia occurred more often in patients who had undergone dysphagia screening than in those who had not, independent from the result of this screening (12.1 vs. $4.7 \%$, respectively; $\mathrm{p}<0.001$ ). Risk to contract pneumonia also increased with late dysphagia screening. The frequency of pneumonia in patients who had not under-

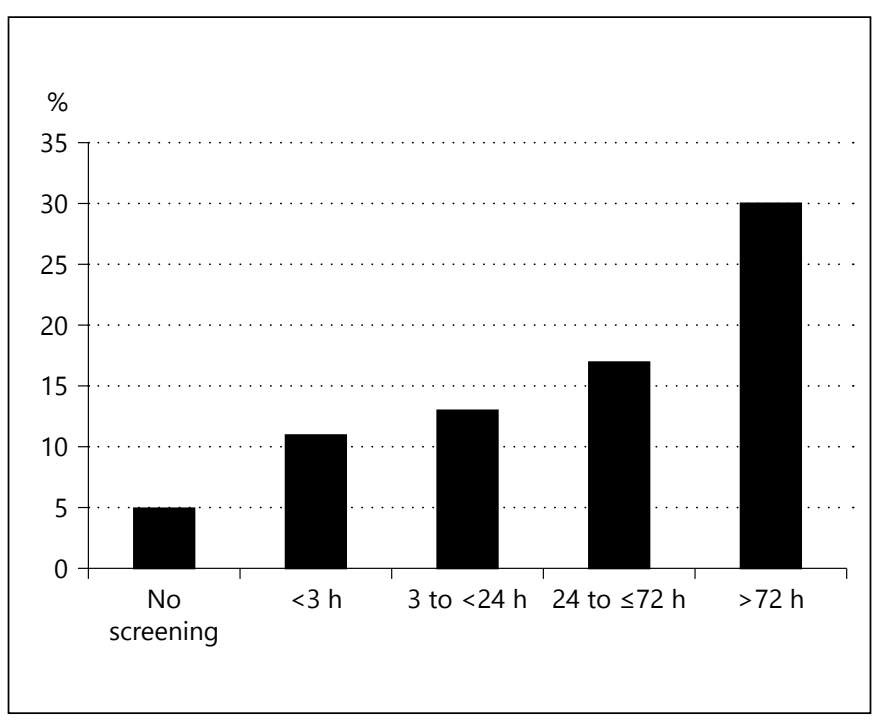

Fig. 2. Pneumonia frequency in relation to assessment time of dysphagia screening. $\mathrm{X}$-axis indicates time point of dysphagia screening, $\mathrm{Y}$-axis frequency of pneumonia.

gone dysphagia screening was $4.7 \%$, whereas the frequency of pneumonia in patients who had undergone dysphagia screening within 3,3 to $<24,24$ to $\leq 72$, and $>72 \mathrm{~h}$ was $10.5,13.2,17.0$, and $29.9 \%$, respectively (fig. 2). A total of 586 patients (4.7\%; 95\% CI 4.3-5.1) died during a mean hospitalization time of 9 days. At discharge from the acute hospital, a disability ( $\mathrm{mRS} \geq 2-5$ ) was present in $60.7 \%$ of all AIS patients who survived. This 
Table 2. Comparison between patients with and without dysphagia

\begin{tabular}{|c|c|c|c|}
\hline \multirow{2}{*}{$\begin{array}{l}\text { Baseline characteristics, therapeutic } \\
\text { procedures and outcomes }\end{array}$} & \multicolumn{2}{|l|}{ Dysphagia } & \multirow[t]{2}{*}{$\mathrm{p}$ value } \\
\hline & no $(\mathrm{n}=9,193)$ & yes $(n=3,083)$ & \\
\hline Age, years, mean \pm SD & $72 \pm 13$ & $76 \pm 12$ & $<0.001$ \\
\hline Median NIHSS score (IQR) & $3(2-5)$ & $13(7-18)$ & $<0.001$ \\
\hline Sex, male & $4,981(54)$ & $1,336(44)$ & $<0.001$ \\
\hline Altered consciousness & $221(2.4)$ & $1,012(33)$ & $<0.001$ \\
\hline Aphasia & $2,145(23)$ & $1,803(59)$ & $<0.001$ \\
\hline Dysarthria & $3,066(34)$ & $2,660(87)$ & $<0.001$ \\
\hline Unilateral weakness & $5,865(64)$ & $2,824(92)$ & $<0.001$ \\
\hline Hypertension & $7,552(83)$ & $2,615(86)$ & $<0.001$ \\
\hline Diabetes mellitus & $2,223(25)$ & $846(28)$ & $<0.001$ \\
\hline Hypercholesterolemia & $5,022(57)$ & $1,455(50)$ & $<0.001$ \\
\hline Previous stroke & $2,405(27)$ & $1,050(35)$ & $<0.001$ \\
\hline Atrial fibrillation & $2,340(26)$ & $1,404(47)$ & $<0.001$ \\
\hline AT before stroke & $3,655(41)$ & $1,448(49)$ & $<0.001$ \\
\hline \multicolumn{4}{|l|}{ Stroke etiology (TOAST) } \\
\hline Large-artery atherosclerosis & $1,996(22)^{\mathrm{a}}$ & $641(21)^{\mathrm{a}}$ & $<0.001$ \\
\hline Cardioembolism & $2,871(32)$ & $1,501(50)$ & \\
\hline Small-artery occlusion & $2,236(25)$ & $268(9)$ & \\
\hline Other determined etiology & $265(3)^{\mathrm{a}}$ & $86(3)^{\mathrm{a}}$ & \\
\hline Undetermined etiology & $1,751(19)^{\mathrm{a}}$ & $567(19)^{\mathrm{a}}$ & \\
\hline Dysphagia screening within $24 \mathrm{~h}$ & $6,151(69)$ & $2,640(89)$ & $<0.001$ \\
\hline Dysphagia screening time, $\mathrm{h}$ & & & $<0.001$ \\
\hline$<3$ & $3,503(39)$ & $1,331(45)$ & \\
\hline 3 to $<24$ & $2,343(26)$ & $1,089(37)$ & \\
\hline 24 to $\leq 72$ & $230(3)$ & $165(6)$ & \\
\hline$>72$ & $75(1)$ & $55(2)$ & \\
\hline Intravenous therapy with rt-PA & $575(6)$ & $561(18)$ & $<0.001$ \\
\hline Oral anticoagulation & $2,657(29)$ & $805(27)$ & 0.006 \\
\hline CEA/stenting & $364(4.5)$ & $60(2.2)$ & 0.001 \\
\hline Antiplatelet treatment & $7,240(80)$ & $2,286(75)$ & 0.001 \\
\hline Thrombosis prophylaxis & $7,675(84)$ & $2,588(85)$ & 0.2 \\
\hline Stroke-related pneumonia & $337(3.7)$ & $917(29.7)$ & $<0.001$ \\
\hline Casa fatality & $106(1.2)$ & $457(14.9)$ & $<0.001$ \\
\hline Disability at discharge $(\mathrm{mRS} \geq 2)$ & $4,628(52)$ & $2,291(89)$ & $<0.001$ \\
\hline Length of hospitalization, mean \pm SD & $9.2 \pm 5$ & $10.5 \pm 7$ & $<0.001$ \\
\hline
\end{tabular}

$\mathrm{AT}=$ Antiplatelet therapy; CEA = carotid thromboendarterectomy. Data are $\mathrm{n}(\%)$ unless otherwise indicated. a Differences after a Bonferroni correction are not significant.

risk of disability was much higher in patients with dysphagia than in those without (89.4 vs. $51.9 \%$, respectively; $\mathrm{p}<0.001)$ as well as in patients who experienced stroke-related pneumonia during hospitalization $(89.9$ vs. $33.3 \%$; $\mathrm{p}<0.001$ ). As shown in table 3 , logistic regression reveals that dysphagia was independently associated with an increased risk of pneumonia $(3.4$; 95\% CI $2.8-$ $4.2 ; \mathrm{p}<0.001$ ), and case fatality (OR 2.8; 95\% CI 2.1-3.7; $\mathrm{p}<0.001)$ during hospitalization as well as disability at discharge (OR 2.0; 95\% CI 1.6-2.3; $\mathrm{p}<0.001$ ). The administration of an EDS within $24 \mathrm{~h}$ of admission appears to be associated with decreased risk of pneumonia during hospitalization (OR 0.68; 95\% CI 0.52-0.89; p = 0.006) and disability at discharge (OR $0.60 ; 95 \%$ CI $0.46-0.77$; $\mathrm{p}<0.001$ ).

At discharge, a total of 7,518 patients or their caregivers agreed to be included in the follow-up evaluation. Data on mortality or disability at 3 months after discharge were recorded for 7,195 patients (96\%) who were included in the follow-up questionnaire. Of these 7,195 patients, 498 patients (6.9\%; 95\% CI 6.3-7.5) died during the 3 -month period after discharge. The 
Table 3. Associated factors with pneumonia, case fatality and disability ( $\mathrm{mRS} \geq 2)$ at discharge in the logistic regressions

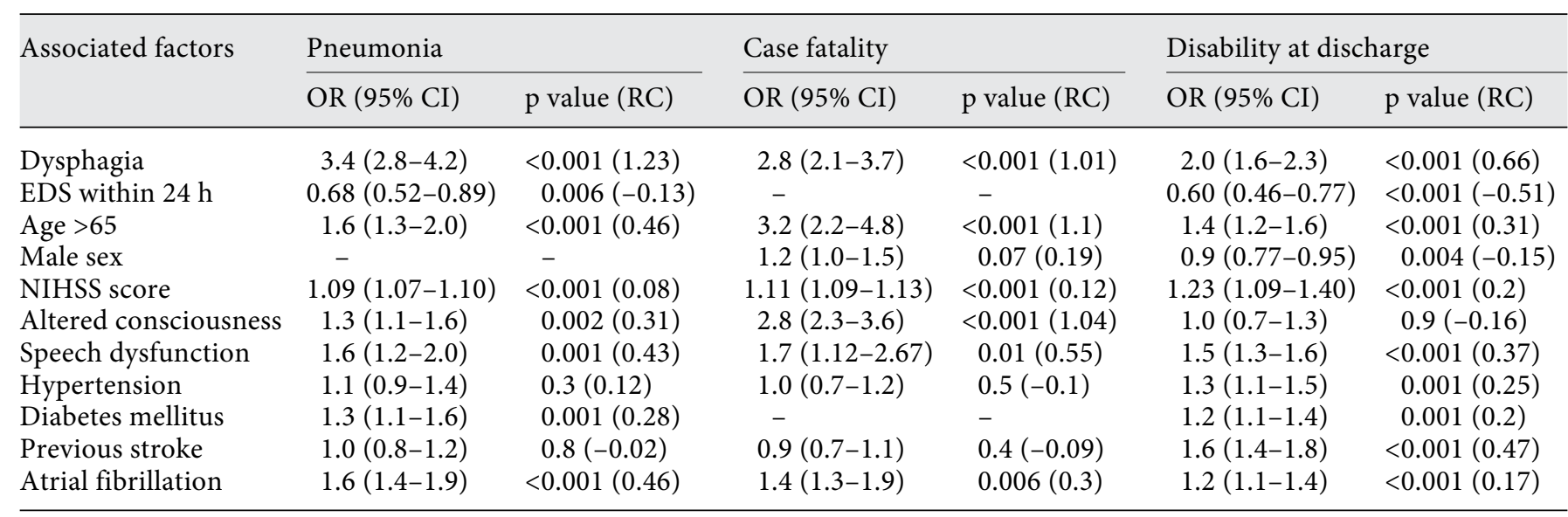

$\mathrm{RC}=$ Regression coefficient.

Table 4. Associated factors with the mortality and the disability (mRS $\geq 2-5$ ) at 3 months after discharge

\begin{tabular}{|c|c|c|c|c|}
\hline \multirow[t]{2}{*}{ Associated factors } & \multicolumn{2}{|c|}{ 3-month mortality } & \multicolumn{2}{|c|}{ 3-month disability } \\
\hline & OR $(95 \%$ CI $)$ & $\mathrm{p}$ value $(\mathrm{RC})$ & OR (95\% CI) & $\mathrm{p}$ value (RC) \\
\hline Dysphagia & $3.2(2.4-4.2)$ & $<0.001(1.15)$ & $2.3(1.8-3.0)$ & $<0.001(0.82)$ \\
\hline EDS within $24 \mathrm{~h}$ & - & - & $0.78(0.51-1.2)$ & $0.2(-0.24)$ \\
\hline Age $>65$ & $2.6(1.8-3.8)$ & $<0.001(0.94)$ & $2.3(1.8-3.0)$ & $<0.001(0.84)$ \\
\hline Male sex & $1.1(0.8-1.3)$ & $0.6(0.05)$ & $0.6(0.54-0.77)$ & $<0.001(-0.44)$ \\
\hline NIHSS score & $1.07(1.04-1.09)$ & $<0.001(0.06)$ & $1.18(1.15-1.21)$ & $<0.001(0.16)$ \\
\hline Altered consciousness & $1.8(1.3-3.4)$ & $<0.001(0.55)$ & $1.3(0.8-2.1)$ & $0.2(0.26)$ \\
\hline Speech dysfunction & $1.0(0.7-1.4)$ & $0.9(0.01)$ & $1.2(1.0-1.5)$ & $0.058(0.21)$ \\
\hline Hypertension & $1.9(1.2-2.9)$ & $0.004(0.62)$ & $1.3(1.0-1.8)$ & $0.04(0.29)$ \\
\hline Diabetes mellitus & - & - & $1.8(1.5-2.3)$ & $<0.001(0.6)$ \\
\hline Previous stroke & $1.2(1.0-1.5)$ & $0.06(0.21)$ & $1.6(1.3-2.0)$ & $<0.001(0.47)$ \\
\hline Atrial fibrillation & $2.0(1.6-2.5)$ & $<0.001(0.69)$ & $1.5(1.2-1.8)$ & $<0.001(0.38)$ \\
\hline
\end{tabular}

$\mathrm{RC}=$ Regression coefficient. Variables with $\mathrm{p}<0.1$ were entered in the logistic regression model.

mortality rate at 3 months after discharge was higher in patients with dysphagia than in those without dysphagia (20.7 vs. $3.4 \%$, respectively; $\mathrm{p}<0.001$ (OR $3.2 ; 95 \%$ CI 2.4-4.2)). There existed an association between ESD within $24 \mathrm{~h}$ and mortality at 3 months. The mortality rate at 3 months of patients who received ESD compared to patients with delayed dysphagia screening was not significantly different ( 8.0 vs. $9.4 \%$, respectively; $\mathrm{p}=0.4$ ). Overall, we found that patients with dysphagia had a higher likelihood of 3-month disability ( $\mathrm{mRS} \geq 2$ 5 ) than patients without dysphagia (74 vs. $25 \%$, respectively; $\mathrm{p}<0.001$ (OR 2.3; 95\% CI 1.8-3.0)). In addition, we found that patients with dysphagia who had undergone EDS within $24 \mathrm{~h}$ had a lower rate of 3-month disability than those who had undergone EDS later ( 40.7 vs. $52 \%$, respectively; $\mathrm{p}=0.003$ ). The logistic regression (table 4) did not show an association between ESD and 3-month disability (OR 0.78; 95\% CI 0.51-1.2; $\mathrm{p}=0.2)$.

\section{Discussion}

In this study, the frequency of dysphagia in patients with AIS is comparable to that of other investigations with a similar design $[11,12]$, but lower than dysphagia
86 
incidence reports from studies that included only patients with brainstem's stroke and used video fluoroscopic swallowing examinations [5, 13-17]. Similar studies that included hemispheric strokes and used mostly clinical dysphagia assessments reported lower dysphagia frequencies [11]. However, in the literature, the incidence of dysphagia after stroke varies greatly, ranging from 25 to $78 \%$, and seems to correlate with the stroke's severity and the type of dysphagia screens techniques [11, 14, 18, 19]. The comparatively low rates of dysphagia as well as stroke-related pneumonia as revealed in this study may be attributed to the design of the study, which included patients with AIS. This is due to the exclusion of patients with hemorrhagic strokes that are generally associated with more severe neurological deficits, particularly altered consciousness and speech dysfunction. In addition, only $74.1 \%$ of patients underwent systematic dysphagia screening. Two main patient groups did not undergo systematic dysphagia screening. One group consisted of patients who showed an immediate improvement in neurological deficits. Patients who did not undergo a dysphagia screening were younger and less affected on the NIHSS score and had lower rates of neurological symptoms at admission. They presented with lower frequencies of vascular risk factors than patients who were screened for dysphagia. In addition to this, patients with minor stroke might be more frequent in this group. In recent years, a gradual change from a time-based definition of transient ischemic attack to a tissue-based one [9, 20-22] has taken place. Hence, more patients with transient neurological symptoms who showed acute infarction by brain imaging were classified as having ischemic stroke according to the proposed definition of transient ischemic attack and stroke $[9,22,23]$. However, this could reflect a selection bias because MRI was not performed among all patients admitted with transient neurological symptoms. Another group of patients who are not screened systematically for dysphagia might be patients with highly impaired consciousness. For those 2 groups of patients, an apparent dysphagia clinical assessment was used instead to determine the frequency of dysphagia.

However, the rate of dysphagia screening in our population is higher than that reported in other investigations reporting that only $50 \%$ of stroke patients are being screened for dysphagia [24]. The occurrence of dysphagia in our study was associated with speech dysfunction and the conscious disturbance of patients. Thus, taken together, patients with dysphagia and altered consciousness may be suffered from more severe stroke than those without dysphagia. According to previous studies [3, 5,
$6,19,25,26]$, we found that patients with dysphagia had a higher risk of suffering from pneumonia after a stroke. This may be explained by the aspiration combined with the effect of immunological alteration and other comorbidities; on the other hand EDS seems to be correlated with a low rate of pneumonia. In this study, we found that EDS within $24 \mathrm{~h}$ of admission reduced the risk of pneumonia noticeably. This could be due to the interventions used after dysphagia detection; namely nasogastric tube feeding among preselected patients with dysphagia and early initiation swallowing therapy to improve cough reflex. Other prophylactic procedures such as the established methods Nil per or status on admission till dysphagia have been excluded, was found to decrease aspiration by targeting swallowing difficulties. These benefits of EDS and early interventions after detection of dysphagia in stroke patients have been shown in previous investigations [27-29]. While this finding is in accordance with the hypothesis that EDS prevents aspiration, we also found that the proportion of dysphagia was higher when dysphagia screening was performed later. On the other hand we have found a strong association between the consciousness status and the time point of dysphagia screening. Thus, the reasons for performing a later dysphagia screening might be that the altered patient's consciousness at admission did not allow for systematic screening, let alone oral food administration. In addition, dysphagia was found to be associated with longer hospitalization and with increased case fatality and disability at discharge; comparable to previous investigations [3033]. On the contrary, we found that the administration of EDS was independently correlated with decreased risk of disability at discharge from acute care hospitals. A correlation between dysphagia and poorer long-term outcomes after stroke has been reported in a previous research [34]. In this study, the rates of mortality and disability were higher in patients with dysphagia at 3 months after discharge. However, an association between EDS within $24 \mathrm{~h}$ and mortality at 3 months was not found in the logistic regression.

This study had several limitations. As mentioned previously, only $74 \%$ of patients underwent systematic dysphagia screening. In addition, approximately one third of our investigation was not included in the 3-month follow-up evaluation. Lastly, the dysphagia screening was performed clinically using water test and clinical examination that are less sensitive than the elaborate video fluoroscopic swallowing examinations.

Despite these limitations, our study has several strengths including its prospectively design, uniform 
study protocol, and standardized stroke care in accordance with the guidelines of the German Stroke Society. Furthermore, the size of the cohort makes this study the largest ever-reported dysphagia study investigating dysphagia and its assessment in patients with ischemic stroke.

\section{Summary}

Our study showed that $25.1 \%$ of patients with AIS have stroke-related dysphagia. The occurrence of dysphagia was strongly associated with longer hospital stay and increased risk of pneumonia and case fatality during hospitalization and disability at discharge as well as with higher rates of mortality and disability at 3 months after discharge. The administration of EDS appears to improve early outcome after stroke.

\section{Acknowledgment and Contributors}

We thank all colleagues treating patients with stroke in Schleswig-Holstein und were involved in the data collection of this study. The following sites participate in the QugSS2 Program: T.F. Münte, MD (Lübeck, director); G. Deuschl, MD (Kiel, director); A. Thie, MD (Itzehoe), J. Hagenah, MD (Heide, head); J. Mudter, MD (Eutin, head); N.L. Sass, MD (Husum, head); C. Schepelmann, MD (Schleswig, head); H.C. Hansen, MD (Neumünster, head); U. Pulkowski, MD (Rendsburg, head); E. Schnieber, MD (Geesthacht, head); K. von Hielcrone, MD and C. Schacherer, MD (Niebuell, heads); G. Hintze, MD (Bad Oldesloe, head); U. Jahnke, MD (Neustadt, head); J.M. Valdueza, MD (Bad Segeberg, head); and M. Nedelmann, MD (Pinneberg, head).

\section{Disclosure Statement}

We declare that we have no conflict of interests.

\section{References}

1 Chamorro A, Meisel A, Planas AM, Urra X, van de Beek D, Veltkamp R: The immunology of acute stroke. Nat Rev Neurol 2012;8:401410.

2 Dirnagl U, Klehmet J, Braun JS, et al: Strokeinduced immunodepression: experimental evidence and clinical relevance. Stroke 2007; 38(2 suppl):770-773.

3 Smithard DG, O’Neill PA, Parks C, Morris J: Complications and outcome after acute stroke. Does dysphagia matter? Stroke 1996; 27:1200-1204.

4 Horner J, Buoyer FG, Alberts MJ, Helms MJ: Dysphagia following brain-stem stroke. Clinical correlates and outcome. Arch Neurol 1991;48:1170-1173.

5 Mann G, Hankey GJ, Cameron D: Swallowing function after stroke: prognosis and prognostic factors at 6 months. Stroke 1999;30:744748.

6 Hannawi Y, Hannawi B, Rao CP, Suarez JI, Bershad EM: Stroke-associated pneumonia: major advances and obstacles. Cerebrovasc Dis 2013;35:430-443.

7 Dennis MS, Lewis SC, Warlow C; FOOD Trial Collaboration: Effect of timing and method of enteral tube feeding for dysphagic stroke patients (food): a multicentre randomised controlled trial. Lancet 2005;365:764-772.

8 Dziewas R, Busse O, Glahn J, et al: [FEES in the stroke unit: recommendations for implementation in the clinical routine]. Nervenarzt 2013;84:705-708

9 Al-Khaled M, Eggers J: MRI findings and stroke risk in TIA patients with different symptom durations. Neurology 2013;80: 1920-1926.

10 Adams HP Jr, Bendixen BH, Kappelle LJ, et al: Classification of subtype of acute ischemic stroke. Definitions for use in a multicenter clinical trial. TOAST. Trial of org 10172 in acute stroke treatment. Stroke 1993;24:35-41.

11 Gottlieb D, Brill S, Khvoles S, Kipnis S, Manevitch D: [Reduction in frequency of pneumonia after stroke, following programmed swallowing evaluation]. Harefuah 1995; 129 : 33-35, 78.

12 Barer DH: The natural history and functional consequences of dysphagia after hemispheric stroke. J Neurol Neurosurg Psychiatry 1989; 52:236-241.

13 Meng NH, Wang TG, Lien IN: Dysphagia in patients with brainstem stroke: incidence and outcome. Am J Phys Med Rehabil 2000;79: 170-175.

14 Daniels SK, Foundas AL: Lesion localization in acute stroke patients with risk of aspiration. J Neuroimaging 1999;9:91-98.

15 Hamdy S, Aziz Q, Rothwell JC, et al: Recovery of swallowing after dysphagic stroke relates to functional reorganization in the intact motor cortex. Gastroenterology 1998;115:11041112.

16 Lim SH, Lieu PK, Phua SY, et al: Accuracy of bedside clinical methods compared with fiberoptic endoscopic examination of swallowing (FEES) in determining the risk of aspiration in acute stroke patients. Dysphagia 2001; $16: 1-6$.

17 Hinds NP, Wiles CM: Assessment of swallowing and referral to speech and language therapists in acute stroke. QJM 1998;91:829-835

18 Martino R, Foley N, Bhogal S, Diamant N, Speechley M, Teasell R: Dysphagia after stroke: incidence, diagnosis, and pulmonary complications. Stroke 2005;36:2756-2763.

19 Gottlieb D, Kipnis M, Sister E, Vardi Y, Brill $\mathrm{S}$ : Validation of the $50 \mathrm{ml} 3$ drinking test for evaluation of post-stroke dysphagia. Disabil Rehabil 1996;18:529-532.

20 Albers GW, Caplan LR, Easton JD, et al: Transient ischemic attack - proposal for a new definition. N Engl J Med 2002;347:17131716.

21 Easton JD, Saver JL, Albers GW, et al: Definition and evaluation of transient ischemic attack: a scientific statement for healthcare professionals from the American Heart Association/American Stroke Association Stroke Council; Council on Cardiovascular Surgery and Anesthesia; Council on Cardiovascular Radiology and Intervention; Council on Cardiovascular Nursing; and the Interdisciplinary Council on Peripheral Vascular Disease. The American Academy of Neurology affirms the value of this statement as an educational tool for neurologists. Stroke 2009;40:22762293.

22 Al-Khaled M, Matthis C, Munte TF, Eggers J: The incidence and clinical predictors of acute infarction in patients with transient ischemic attack using MRI including DWI. Neuroradiology 2013;55:157-163.

23 Sacco RL, Kasner SE, Broderick JP, et al: An updated definition of stroke for the 21st century: a statement for healthcare professionals from the American Heart Association/ American Stroke Association. Stroke 2013;44: 2064-2089.

24 Martino R, Martin RE, Black S: Dysphagia after stroke and its management. CMAJ 2012; 184:1127-1128.

25 Sala R, Munto MJ, de la Calle J, et al: [Swallowing changes in cerebrovascular accidents: incidence, natural history, and repercussions on the nutritional status, morbidity, and mortality]. Rev Neurol 1998;27:759-766. 
26 Holas MA, DePippo KL, Reding MJ: Aspiration and relative risk of medical complications following stroke. Arch Neurol 1994;51: 1051-1053.

27 Carnaby G, Hankey GJ, Pizzi J: Behavioural intervention for dysphagia in acute stroke: a randomised controlled trial. Lancet Neurol 2006;5:31-37.

28 DePippo KL, Holas MA, Reding MJ, Mandel FS, Lesser ML: Dysphagia therapy following stroke: a controlled trial. Neurology 1994;44: 1655-1660.
29 Crary MA, Carnaby Mann GD, Groher ME, Helseth E: Functional benefits of dysphagia therapy using adjunctive sEMG biofeedback. Dysphagia 2004;19:160-164.

30 Guyomard V, Fulcher RA, Redmayne O, Metcalf AK, Potter JF, Myint PK: Effect of dysphasia and dysphagia on inpatient mortality and hospital length of stay: a database study. J Am Geriatr Soc 2009;57:2101-2106.

31 Paciaroni M, Mazzotta G, Corea F, et al: Dysphagia following stroke. Eur Neurol 2004;51: 162-167.
32 Maeshima S, Osawa A, Miyazaki Y, et al: Influence of dysphagia on short-term outcome in patients with acute stroke. Am J Phys Med Rehabil 2011;90:316-320.

33 Maeshima S, Osawa A, Hayashi T, Tanahashi $\mathrm{N}$ : Factors associated with prognosis of eating and swallowing disability after stroke: a study from a community-based stroke care system. J Stroke Cerebrovasc Dis 2013;22:926-930.e1.

34 Smithard DG, Smeeton NC, Wolfe CD: Longterm outcome after stroke: does dysphagia matter? Age Ageing 2007;36:90-94. 